\section{VOUS CHERCHEZ DES MANUELS DE REDACTION?}

Joan E. Pavelich, UnIversitê de Sherbrooke

Il est un falt évident que les livres expliquant 1a rédaction technique sont plutôt publiés en anglais qu'en français. Néanmoins, de bons manuels frança1s existent. La présente bibllographie est une liste des mellleurs ouvrages trouvés sur les rayons ou dans les flchlers de la blbliothèque montréalałse de l'office de la langue française. Chaque entrée bibllographique est sufvie d'une description des objectifs du livre, tirée du volume lui-même, ou de l'avis d'un critique sur le volume en question. Nécessairement, cette blbllographie n'est pas une liste exhaustive des manuels disponibles en françals. Si vous en connsissez d'autres, aldez nos collègues en envoyant une description au directeur de Technostyle.

Eléments de bibliographie

\section{Techniques de la communication}

Bar1l, Denis, et Jean Guillet. Techniques de l'expression écrite et orale, 4e éd., Paris, Edittons Sirey, 1978, 2 vol., 553 p.

"Le premfer volume propose une initiation aux techniques de base de la communication orale et écrite. Il enseigne à élaborer et transmettre l'information, et a la recevolr aussi. Le second volume concerne les techniques plus complexes du rapport, des écrits et interventions orales plus amples et plus nuancées. Il forme à l'expression du jugement et 11 a surtout pour but de mettre l'expression au service de l'action et de la persuasion." (p.10)

Ducassê, Plerre. Formation intellectuelle et méthodes d'expression, Paris, Dunod, 1967, 2 vol., 300 p.

Les techniques de base de la communication, décrites dans le premier volume, font 1 'objet des applications concrètes et des travaux pratiques dans le second volume.

Jeoffrey-Faggianell1, Pierrette, et Louis-Robert Plazolles. Techniques de l'expression et de 1a communication. Paris, Nathan, 1979, 144p.

Sujets traftés: la recherche des informations, le traltement des informations dans les messages de structure simple et complexe et 1 'échange des informations.

Vanoye, Francis. Expresston communication, Paris, Armand Colin, $1973,242 \mathrm{p}$.

"Cet ouvrage s'adresse à tous ceux qui enseignent et étudient les Techniques d'Expression dans:

- les Instituts Universitafres de Technologie;

- le cadre de la licence d'Etudes Pratiques;

- les classes de préparation aux grandes Ecoles.

I1 rendra également des services à tous ceux qui, dans les cadres scolaire, universitaire ou professionnel, ont à

s'exprimer et communiquer oralement ou par écrit." (p.5)

\section{Correspondance et rédaction administratives}

Audry, M. et J. Roumagnac. Précls de rédaction de rapports, comptes rendus, procès-verbaux, notes et instructions, Paris, Editions Foucher, 1969, 167 p.

Bousquie, G. Comment rédiger vos rapports, 9e éd., Paris, Entreprise moderne d'édition, 1973, $150 \mathrm{p}$.

Ouvrage recommandé par la Direction du perfectionnement de la Commission de la fonction publique du Canada et par l'office de la langue française.

Cafolet-Laganière, Hểène. Le frança1s au bureau, 2e êd. rev. et augm., Québec, Editeur officiel du Québec, 1982, 197 p. 
"La première partie traite des règles et des usages de la correspondance, comme la présentation de la lettre et du rapport, 1 'adressage de 1 'enveloppe; divers exemples de rédaction administrative et des modèles d'adresses complètent et lllustrent ce chapitre. La deuxlème partie, portant sur la grammafre et le vocabulaire, relève les fautes les plus courantes relatives au code grammatical et au lexique usuel du français au bureau. De plus, un vocabulaire technique réunit quelque deux cents termes parmi les plus utilisés, concernant 1 'ameublement et le matériel de bureau; les définitions ainsi que les équivalents angla1s ont été omis et remplacés par des 1llustrations. Une troisième partie tralte de quelques problèmes de la langue écrite, par exemple l'emplo1 de la majuscule, des sigles, symboles et abréviations et des signes de ponctuation. Quelques conseils relatifs au protocole téléphonique terminent cette dernière partie." (p.11)

Catherine, Robert. Le style administratif, 9e éd., Paris, Albin Michel, 1979, $171 \mathrm{p}$.

Un manuel françals que nos rédacteurs administratifs consultent souvent.

Clas, André, et Paul A. Horguelin. Le français, langue des affatres, 2e êd., Montréal, McGraw-H111, 1979, 391 p.

La deuxième édition de ce manuel très utile est maintenant sortie. Accompagné d'un cahier d'exercices.

Desseaux, Plerre. Rapports et comptes-rendus, Se éd., France, Editions Hommes et Techniques, 1970, 117 p.

"Ecrits par des auteurs qui sont des praticiens de la formation dans les entreprises ou qui enseignent en milieu industriel, les ouvrages [de 1a collection 'Formation active'] sont conçus en fonction des besolns intellectuels et du mode de pensée de ce public, essentiellement Inductif et concret. Evitant la présentation scolalre sans sacrifler le sérieux, les matières sont enseignées sous une forme seml-romancée où des personnages de la vie courante sont mis en situation, confrontés avec leurs problèmes. D'une présentation agréable par les fllustrations et d'une lecture facile grâce au choix des caractères, aux sommalres et résumés qui donnent le fil des 1 dées, ces manuels sont directement abordables par un public non inftié ou peu habituê à 1ire." (pp. 5-6)

Direction du perfectionnement, Comission de la fonction public du Canada. Guide de rédaction administrative: préface, méthodologle et explication, table des matières, Ottawa, Information Canada, 1974, 100 p.

"Le présent document est un instrument de référence qui vise à fournir les renseignements et la documentation nécessalre pour la rédaction de documents administratifs françals à la Fonction publique...

Ce document a été conçu d'après la synthése de nombreux ouvrages qui traitent du sujet et d'après de nombreux documents administratifs en usage. Nous avons essayé de concilier ce que pense le linguiste, ce qu'utilise l'administrateur et ce qui, selon nous, est fldèle à la mentalité canadienne-française." (p.1)

Gandouin, Jacques. Correspondance et rédaction administratives, 7e éd., Paris, Armand Col1n, 1980, 359 p.

Considéré "un ouvrage essent1el" par Cajolet-Laganière.

Gaubert, Gaston. Comment rédiger les documents professionnels, 2e éd., Paris, Editions Eyrolles, 1977, 122 p. 
"Nous avons tenté d'exposer clafrement et de façon concise les règles à respecter en ce qui concerne la présentation et la rédaction des documents établis dans 1 'exercice des activités professionnelles. .

Nous avons pensé aux besoins de nos élèves ingénieurs et des Jeunes cadres qui éprouvent souvent, en début de carrière, des hésitations quant à la façon de présenter et de rédiger les écrits qui leur sont demandés." (p.1)

Mauger, Gaston, et Jacqueline Charron. Le françals commercial, 6e êd., Paris, Larousse, 1971, 312 p.

P1llaud, Marcel, et Edouard Leury. Rédaction administrative, Ottawa, Commission de la fonction publique du Canada, Bureau des langues, 1971, 2 vol.

Conçu par et pour les fonctionnatres fédéraux, ce volume est le résultat des cours de rédaction donnés pendant plusieurs années par la Commission.

Van Colllie-fremblay, Brigitte. Guide pratique de correspondance et de rédaction, Québec, Editeur officiel du Québec, 1976, $201 \mathrm{p}$.

"Le présent guide, conçu pour le personnel de la Régie des rentes du Québec, vise à réunir en un seul ouvrage des réponses aux nombreuses questions quil se posent lors de la rédaction et la transcription de lettres, de rapports ou de formules." (p.vi11)

Wackerman, G. et A. Wilhelm. Initiation à la technique du rapport, 2e éd. rev. et augm., Paris, Dunod, 1974, 144 p.

Le manuel est recommandé par la Commission de la fonction publique du Canada et par Cajolet-Lagantère.
Recherche et publication

Desha1s, Bruno, et.al. Comment rédiger un rapport de recherche, Se éd., Montréal, Leméac, 1974, 72 p.

"La présente brochure traite uniquement de la présentation formelle du rapport de recherche. Limitée à l'aspect technique, elle vise cependant à promouvolr l'esprit de clạrté qui doit animer la présentation de tout travall de recherche sérfeux. Le premier chapitre, mises en pages, Indique comment organiser les diverses parties du rapport et comment donner à chacune une allure solgnée et dégagée. Les trois chapitres qui suivent, blbliographie, c1tations, références et notes, concernent la présentation des sources utilisées au cours des recherches et pendant la rédaction du rapport." (p.vi1)

Groupe de rraval1 sur la rédaction technique de l'Ecole polytechnique de Montréal. Guide de rédaction. Projet de fin d'études. Montréal, Ecole polytechn1que de Montréal, 1981, 44 p.

"Le but de ce cahter est d'abord d'aider l'étudiant finissant à 1 'Ecole Polytechnique de Montréal (EPM) dans la rédaction du rapport de son profet de fin d'études. On se propose d'atteindre ce but en respectant les règles communément admises pour la présentation de rapports techniques dans la pratique de 1 'ingénierte.

Ce cahler se veut aussi un guide pour la rédaction de tout rapport un tant soit peu élaboré que le jeune ingênleur devra écrire au début de sa carrière." (p.1)

Poirier, Léandre, o.f.m. Au service de nos écrivalns. Directives pratiques pour publications, 3e éd., rev., Montréal, Fides, $1964,193 \mathrm{p}$. 
Selon Deshaies et. al.,"Tous ceux qui doivent écrire - les étudiants en particulier - trouveront ce volume très précleux. D'ailleurs, depuis la première êdition en 1943, ces Directives pratiques ont rendu de grands services. Les consells judicleux de l'auteur s'appuient sur une longue expérience dans le domaine du travall intellectuel. Le chapitre III de la première partle traite de 'La présentation scient1fique' (pp. 75-105) du rapport de recherche." (p.5)

Vinet, Bernard. La bibliothèque, Instrument de travail 1, Montréal, Centre de Psychologie et de Pédagogle, 1962, 130 p.

Hautement recommandé par les bibllothécaires de l'office de la langue française. Selon Deshaies et.al., "Ce livre sera utile à l'étudiant qui n'a pas été inftié à faire de la recherche dans une bibliothèque. L'étudiant apprendra a chercher les informations bibliographiques qui lu1 seront Indispensables ensuite pour indiquer ses sources dans la blbllographie ou dans les références." (p.5)

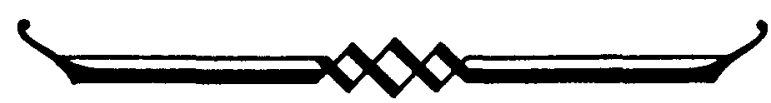

